\title{
Direction-Changing and Direction-Fixed Positional Nystagmus in Patients With Vestibular Neuritis and Meniere Disease
}

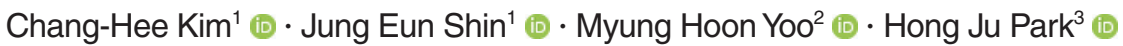 \\ ${ }^{1}$ Department of Otorhinolaryngology-Head and Neck Surgery, Konkuk University Medical Center, Konkuk University School of Medicine, Seoul; \\ ${ }^{2}$ Department of Otorhinolaryngology-Head and Neck Surgery, College of Medicine, Kyungpook National University, Daegu; \\ ${ }^{3}$ Department of Otorhinolaryngology-Head and Neck Surgery, Asan Medical Center, University of Ulsan College of Medicine, Seoul, Korea
}

Objectives. Direction-changing positional nystagmus (PN) was considered to indicate the presence of benign paroxysmal positional vertigo involving lateral semicircular canal in most cases. We investigated the incidence of PN on the supine head-roll test and compared the characteristics of nystagmus in patients with vestibular neuritis (VN) and Meniere disease (MD).

Methods. A retrospective review of patients, who were diagnosed with unilateral VN or unilateral definite MD between September 2005 and November 2011, was conducted. Sixty-five VN patients and 65 MD patients were enrolled. Eye movements were recorded for 30-60 seconds at the positions of sitting, head roll to the right, and head roll to the left, and maximum slow-phase eye velocity was calculated. PN was classified as direction-fixed (paretic or recovery) and direction-changing (geotropic or apogeotropic).

Results. Spontaneous nystagmus was observed in 57 patients (87\%, the slow-phase eye velocity of $7 \% / \mathrm{sec} \pm 5 \% \mathrm{sec}$ ) with acute VN, $39(60 \%, 2 \% \mathrm{sec} \pm 1 \% \mathrm{sec})$ with follow-up VN, and $32(49 \%, 2 \% \mathrm{sec} \pm 2 \% \mathrm{sec})$ with MD. Direction-fixed PN was the most common type. Direction-fixed paretic type was most common in acute VN (80\%) and follow-up VN (42\%), and direction-fixed recovery type was most common in MD (31\%). Paretic type was significantly more common in acute $\mathrm{VN}(80 \%)$ than in follow-up VN $(42 \%)$ and $\mathrm{MD}(26 \%)$, and the recovery type was significantly more common in $\mathrm{MD}(31 \%)$ than in acute VN (3\%) and follow-up VN (14\%). Direction-changing PN was more common in MD (22\%), followed by follow-up VN (14\%) and acuteVN (9\%).

Conclusion. Though direction-fixed paretic PN was most common in VN and MD patients, direction-changing PN could be observed in a few patients $(9 \%-20 \%)$ with peripheral vestibular disorders regardless of the duration from the onset of dizziness, suggesting the presence of otolith-related dizziness.

Keywords. Vestibular Neuritis; Meniere Disease; Benign Paroxysmal Positional Vertigo; Nystagmus; Positional

\section{INTRODUCTION}

- Received January 10, 2018

Revised October 4, 2018

Accepted October 16, 2018

- Corresponding author: Hong Ju Park

Department of Otorhinolaryngology-Head and Neck Surgery, Asan Medical Center, University of Ulsan College of Medicine, 88 Olympic-ro 43-gil, Songpa-gu, Seoul 05505, Korea

Tel: +82-2-3010-3700, Fax: +82-2-489-2773

E-mail: dzness@hotmail.com

\begin{abstract}
Vestibular neuritis $(\mathrm{VN})$, which is one of the most common causes of acute spontaneous vertigo, is characterized by sudden onset of peripheral vertigo without associated hearing loss [1]. Meniere disease (MD) is a common inner ear disorder that causes hearing symptoms and recurrent vertigo attacks $[2,3]$. Placing a patient with a vestibular dysfunction caused by VN or MD into various positions may induce a direction-fixed or direction-changing positional nystagmus (PN). When the restoration
\end{abstract}

Copyright @ 2019 by Korean Society of Otorhinolaryngology-Head and Neck Surgery.

This is an open-access article distributed under the terms of the Creative Commons Attribution Non-Commercial License (http://creativecommons.org/licenses/by-nc/4.0)

which permits unrestricted non-commercial use, distribution, and reproduction in any medium, provided the original work is properly cited. 
of vestibular tone occurs with static compensation, placing the patient into different positions, provoking neural firing from the labyrinths, may cause the reappearance or augmentation of spontaneous nystagmus (SN). This PN would be expected to be direction-fixed and horizontal with the fast component of the nystagmus directed towards the intact ear. If otolith particles involve the lateral semicircular canal (LSCC), the detection of a direction-changing PN (geotropic or apogeotropic) can be used to diagnose the presence of a lateral canal benign paroxysmal positional vertigo (BPPV). Vigorous SN, which is commonly observed during the acute stage of $\mathrm{VN}$ or in some MD patients with recent vertigo attacks, may mask the presence of PN when it occurs concomitantly with BPPV.

The PN in patients with VN and MD has not been reported thoroughly so far. We wanted to investigate the incidence of a direction-fixed or direction-changing (geotropic or apogeotropic) PN during the supine head-roll test systematically in patients with $\mathrm{VN}$ and $\mathrm{MD}$, and to compare the characteristics of nystagmus in those patients.

\section{MATERIALS AND METHODS}

\section{Patients}

A retrospective review of patients, who were diagnosed with unilateral VN or unilateral definite MD between September 2005 and November 2011, was conducted. We enrolled 65 patients with unilateral VN (male:female, 33:32; age range, 15 to 73 years). The patients with VN met the clinical diagnostic criteria for $\mathrm{VN}$, including sudden onset of vertigo for more than 1 day with unidirectional spontaneous horizontal nystagmus, absence of other auditory and neurologic findings, reduced canal paresis (CP) calculated by Jongkees' formula ( $\mathrm{CP} \geq 25 \%$ ), and no previous history of vertigo or other neurotologic diseases. Brain magnetic resonance imaging (MRI) did not reveal acute infarction or other acute/chronic brain lesions, including cerebellopontine angle tumors, in any of the patients. Patients with interaural differences of more than $15 \mathrm{~dB}$ were excluded from the study. Vestibular tests including the caloric test and PN test were performed during the acute stage as soon as the patient's symp-

\section{H I $G$ G H L I}

- Direction-changing positional nystagmus (PN) indicates benign paroxysmal positional vertigo in most cases.

- Direction-fixed PN can be towards (paretic) or against (recovery) the intact ear.

- Direction-fixed paretic PN was most common in vestibular neuritis, and direction-fixed recovery PN in Meniere disease.

- Direction-changing PN was observed in 9\%-22\% of peripheral dizziness. toms permitted (within 8 days after the onset of vertigo), and the follow-up tests were performed about 2 months after the onset of vertigo.

We also retrospectively evaluated 65 patients (male:female, 28:37; age, $37 \pm 11$ years) with definite unilateral $M D$ as defined by the 1995 criteria of the American Academy of Otolaryngology-Head and Neck Surgery. The involved side was the right side in 23 patients and the left side in 42 patients. None of the patients were taking medication that affects the central nervous system, and none had positive findings from neurological examinations or MRI. Vestibular tests were performed during an interictal period, usually within 10 days after the first visit to the clinic.

This study protocol was approved in the Institutional Review Board of the Konkuk University Medical Center (IRB No. Headroll KUH1110033).

\section{Head-roll test}

The slow-phase velocity (SPV) of SN was determined as the average of the three fastest velocities measured during a 10-second time window using a video-based system (ChartrVNG, ICS Medical, Schaumburg, IL, USA), while the subject was sitting in the dark. Then, the supine head-roll test was performed by turning the patient's head to the right or left in the supine position, and eye movements were recorded for 30-60 seconds.

We investigated the characteristics of PN and the incidence of PN in the supine head-roll test. For clarity, PN during head roll was classified into direction-fixed and direction-changing PN: (1) paretic direction-fixed type when the direction of PN was towards the healthy side in at least one side in the supine headroll test, (2) recovery direction-fixed type when the direction of PN was towards the lesioned side in at least one side in the supine head-roll test, (3) geotropic direction-changing type when geotropic PN was observed in the supine head-roll test, (4) apogeotropic direction-changing type when apogeotropic PN was observed in the supine head-roll test, and (5) no nystagmus when no position nystagmus was observed in head roll to both sides. The lowest value of the SPV of $1 \%$ sec or greater was considered as the presence of PN.

\section{Caloric test}

The bithermal caloric test was performed while recording eye movements using a video-based system (ChartrVNG, ICS Medical). Each ear was irrigated with a constant flow of water at alternate temperatures of $30^{\circ} \mathrm{C}$ and $44^{\circ} \mathrm{C}$. The maximum SPV of nystagmus was measured following irrigation, and Jongkees' formula was used to determine CP.A CP of $25 \%$ or more was considered abnormal. During procedures, patients were instructed to open their eyes widely while wearing infrared goggles in a dark room.

\section{Data analysis}

Data were expressed as the mean and standard deviation. Categorical variables were evaluated using Fisher exact test using 
SPSS ver. 18.0 (SPSS Inc., Chicago, IL, USA). All values of $P<0.05$ were considered significant.

\section{RESULTS}

SN was observed in 57 (87\%, the slow-phase eye velocity of $7 \% \mathrm{sec} \pm 5 \% \mathrm{sec}), 39(60 \%, 2 \% \mathrm{sec} \pm 1 \% \mathrm{sec})$, and 32 patients $(49 \%$, $2 \% \mathrm{sec} \pm 2 \% \mathrm{sec}$ ) in acute $\mathrm{VN}$, follow-up VN, and $\mathrm{MD}$, respectively. Although the direction of SN was towards the intact side in most patients, two patients (4\%) with acute VN and six patients $(15 \%)$ at the follow-up stage showed SN towards the lesioned side. In MD, 13 patients (41\%) showed SN directed towards the lesioned side, and 19 patients (59\%) showed SN directed towards the intact side. CP values in the caloric test were $62 \% \pm 19 \%$ and $46 \% \pm 32 \%$ in acute $\mathrm{VN}$ and follow-up VN, respectively. Sixteen patients $(25 \%)$ showed normalization of CP at the follow-up stage. In MD, 38 patients (58\%) showed abnormal CP.

In acute $\mathrm{VN}, 52$ patients $(80 \%)$ showed the paretic directionfixed type, and two (3\%) showed the recovery direction-fixed type. Six patients $(9 \%)$ showed direction-changing PN, of which three showed the geotropic direction-changing type and three showed the apogeotropic direction-changing type (Table 1). In patients with direction-fixed $\mathrm{PN}$, the direction of $\mathrm{SN}$, if present, was same as that of PN except one patient with recovery-type $\mathrm{PN}$ of which the direction was opposite to that of $\mathrm{SN}$, and the SPV was only $1 \%$ sec in both SN and PN. In five of 65 patients $(8 \%)$, no PN was observed (Table 1$)$. In the geotropic type $(n=3)$, the nystagmus on head roll to the lesioned side was stronger than or similar to that on the healthy side in two $(67 \%)$ patients (Table 2). In the apogeotropic type $(n=3)$, stronger or similar nystagmus was observed when the patient's head was turned to the healthy side in all patients (Table 2). Among three patients with geotropic type, one (patient No. 3 in Table 2) still showed geotropic type at follow-up stage, one showed paretic-type PN, and one showed no PN at follow-up stage. Among three patients with apogeotropic type, one (patient No. 5 in Table 2) still showed apogeotropic type at follow-up stage, and two showed recoverytype $\mathrm{PN}$ at follow-up stage.

In follow-up VN, the paretic direction-fixed type was observed in 27 patients $(42 \%)$ and the recovery direction-fixed type in nine (14\%) (Table 1). In patients with direction-fixed PN, the direction of $\mathrm{PN}$ was same as that of $\mathrm{SN}$ in all patients except one who showed recovery-type PN, and the SPV was only $1 \%$ sec in both SN and PN. Direction-changing PN was observed in nine patients $(14 \%)$, and the geotropic direction-changing type (six of nine) was more common than the apogeotropic directionchanging type (three of nine). Among six patients with geotropic type, one (patient No. 3 in Table 2) also showed geotropic type at acute stage, two showed recovery-type $\mathrm{PN}$, and three showed paretic-type PN at acute stage. Among three patients with apogeotropic type, one (patient No. 7 in Table 2) also showed apogeotropic type at acute stage, and two showed paretic-type PN at acute stage. In the geotropic direction-changing type $(n=6)$, the nystagmus on head roll to the lesioned side was stronger than or similar to that on the healthy side in five patients $(83 \%)$ (Table 2 ). In the apogeotropic type ( $n=3)$, a stronger or similar nystagmus was observed when the patient's head was turned to the healthy side in two patients $(67 \%)$. In 20 of 65 patients (31\%), no PN was observed on either right or left head roll (Table 1).

In $\mathrm{MD}$, the paretic direction-fixed type was observed in 17 patients $(26 \%)$, and the recovery direction-fixed type was observed in 20 (31\%) (Table 1). The direction of PN was same as that of SN in all patients with direction-fixed PN. Fourteen patients $(22 \%)$ showed direction-changing PN, of which seven showed the geotropic direction-changing type and seven showed the apogeotropic direction-changing type (Table 1). In the geotropic direction-changing type $(n=7)$, the nystagmus on head roll to the lesioned side was stronger than or similar to that on the healthy side in five (71\%) patients (Table 2). In the apogeotropic direction-changing type $(\mathrm{n}=7)$, a stronger or similar nystagmus was observed when the patient's head was turned to the healthy side in four patients (57\%).

The paretic type in acute VN (80\%) and follow-up VN (42\%)

Table 1. Types and incidence of positional nystagmus in the supine head-roll test in patients with VN in the acute stage and at follow-up, and MD

\begin{tabular}{|c|c|c|c|c|c|}
\hline \multirow{2}{*}{ Variable } & \multicolumn{2}{|c|}{ Direction-fixed } & \multicolumn{2}{|c|}{ Direction-changing } & \multirow{2}{*}{$\begin{array}{c}\text { No } \\
\text { nystagmus }\end{array}$} \\
\hline & Paretic type & Recovery type & Geotropic type & Apogeotropic type & \\
\hline Acute VN $(n=65)$ & $52(80)$ & $2(3)$ & $3(5)$ & $3(5)$ & $5(7)$ \\
\hline$S N+(n=57)$ & $51(90)$ & $2(3)$ & $2(3)$ & $1(2)$ & $1(2)$ \\
\hline$S N-(n=8)$ & $1(13)$ & 0 & $1(13)$ & $2(25)$ & $4(49)$ \\
\hline Follow-up VN $(n=65)$ & $27(42)$ & $9(14)$ & $6(9)$ & $3(5)$ & $20(30)$ \\
\hline $\mathrm{SN}+(\mathrm{n}=38)$ & $24(63)$ & $6(16)$ & $3(8)$ & $1(3)$ & $4(10)$ \\
\hline$S N-(n=27)$ & $3(11)$ & $3(11)$ & $3(11)$ & $2(8)$ & $16(59)$ \\
\hline $\mathrm{MD}(\mathrm{n}=65)$ & $17(26)$ & $20(31)$ & $7(11)$ & $7(11)$ & $14(21)$ \\
\hline $\mathrm{SN}+(\mathrm{n}=32)$ & $12(38)$ & $12(38)$ & $2(6)$ & $2(6)$ & $4(12)$ \\
\hline $\mathrm{SN}-(\mathrm{n}=33)$ & $5(15)$ & $8(24)$ & $5(15)$ & $5(15)$ & $10(31)$ \\
\hline
\end{tabular}

Values are presented as number (\%).

VN, vestibular neuritis; MD, Meniere disease; SN, spontaneous nystagmus. 
Table 2. Clinical characteristics and findings of the head-roll test in patients showing direction-changing PN

\begin{tabular}{|c|c|c|c|c|c|c|c|c|c|}
\hline Variable & No. & Age/sex & Side & $\begin{array}{l}\mathrm{CP} \\
(\%)\end{array}$ & Direction & $\begin{array}{l}\text { SPV_L } \\
(\% / \mathrm{sec})\end{array}$ & $\begin{array}{c}\mathrm{SN} \\
(\% / \mathrm{sec})\end{array}$ & $\begin{array}{l}\text { SPV_R } \\
(\% / \mathrm{sec})\end{array}$ & $\begin{array}{l}\text { Possible } \\
\text { lesion side }\end{array}$ \\
\hline \multirow[t]{6}{*}{ Acute VN } & 1 & $63 / F$ & Left & 84 & Geotropic & 2 & -3 & -10 & Contra \\
\hline & 2 & $52 / \mathrm{M}$ & Left & 48 & Geotropic & 1 & 0 & -1 & - \\
\hline & 3 & $69 / F$ & Left & 55 & Geotropic & 9 & -2 & -6 & Ipsi \\
\hline & 4 & $43 / F$ & Left & 51 & Apogeotropic & -3 & -2 & 1 & Ipsi \\
\hline & 5 & $54 / F$ & Left & 44 & Apogeotropic & -1 & 0 & 1 & - \\
\hline & 6 & $50 / \mathrm{M}$ & Right & 67 & Apogeotropic & -15 & 0 & 6 & Ipsi \\
\hline \multirow[t]{9}{*}{ Follow-up VN } & 1 & $45 / M$ & Left & 86 & Geotropic & 3 & -2 & -4 & Ipsi \\
\hline & 2 & $50 / \mathrm{M}$ & Left & 11 & Geotropic & 1 & -2 & -2 & Ipsi \\
\hline & 3 & $69 / F$ & Left & 38 & Geotropic & 2 & -1 & -3 & Ipsi \\
\hline & 4 & $50 / F$ & Left & 26 & Geotropic & 1 & 0 & -1 & - \\
\hline & 5 & $59 / M$ & Right & 64 & Geotropic & 4 & 0 & -3 & Contra \\
\hline & 6 & $34 / \mathrm{M}$ & Right & 7 & Geotropic & 1 & 0 & -1 & - \\
\hline & 7 & $54 / F$ & Left & 51 & Apogeotropic & -2 & 0 & 3 & Ipsi \\
\hline & 8 & $37 / F$ & Right & 59 & Apogeotropic & -2 & 0 & 1 & Ipsi \\
\hline & 9 & $47 / M$ & Right & 94 & Apogeotropic & -4 & 2 & 9 & Contra \\
\hline \multirow[t]{14}{*}{ MD } & 1 & $21 / F$ & Left & 20 & Geotropic & 3 & 0 & -5 & Contra \\
\hline & 2 & $36 / \mathrm{M}$ & Left & 10 & Geotropic & 2 & 0 & -2 & - \\
\hline & 3 & $59 / \mathrm{M}$ & Left & 9 & Geotropic & 3 & -1 & -3 & Ipsi \\
\hline & 4 & $57 / F$ & Left & 12 & Geotropic & 1 & -1 & -1 & Ipsi \\
\hline & 5 & $61 / F$ & Left & 27 & Geotropic & 1 & 0 & -1 & - \\
\hline & 6 & $40 / F$ & Right & 5 & Geotropic & 2 & 0 & -3 & Ipsi \\
\hline & 7 & $44 / \mathrm{M}$ & Right & 50 & Geotropic & 6 & 0 & -2 & Contra \\
\hline & 8 & $43 / \mathrm{M}$ & Left & 13 & Apogeotropic & -1 & 0 & 1 & - \\
\hline & 9 & $65 / \mathrm{M}$ & Left & 51 & Apogeotropic & -12 & -1 & 8 & Contra \\
\hline & 10 & $32 / F$ & Right & 23 & Apogeotropic & -1 & 1 & 6 & Contra \\
\hline & 11 & $36 / F$ & Right & 26 & Apogeotropic & -1 & 0 & 1 & - \\
\hline & 12 & $28 / \mathrm{M}$ & Right & 20 & Apogeotropic & -1 & 0 & 1 & - \\
\hline & 13 & $36 / F$ & Right & 38 & Apogeotropic & -3 & 0 & 2 & Ipsi \\
\hline & 14 & $22 / M$ & Right & 13 & Apogeotropic & -3 & 0 & 4 & Contra \\
\hline
\end{tabular}

PN, positional nystagmus; CP, canal paresis; SPV_L, slow-phase velocity when the head roll to the left side; SN, spontaneous nystagmus; SPV_R, slowphase velocity when the head roll to the right side; VN, vestibular neuritis; F, female; Contra, contralateral to the lesioned side; M, male; Ipsi, ipsilateral to the lesioned side; MD, Meniere disease.

was the most common type, and the recovery type was the most common in $\mathrm{MD}(31 \%)$. The paretic type was significantly more common in acute VN $(80 \%)$ than in follow-up VN $(42 \%)$ and MD (26\%), and the recovery type was significantly more common in MD (31\%) than in acute VN $(3 \%)$ and follow-up VN $(14 \%)$. The incidence of direction-changing PN in the supine head-roll test was the highest in MD $(22 \%)$, followed by followup VN (14\%) and acute VN (9\%), but a statistically significant difference was not observed $(P>0.05$, Fisher exact test).

\section{DISCUSSION}

In this study, the paretic direction-fixed PN in acute VN $(80 \%)$ and follow-up VN (42\%) was the most common type, and the recovery direction-fixed PN was most common in MD (31\%). When placing the patient into different positions, which provoke neural firing from the labyrinth, may result in the reappearance or augmentation of SN. This PN would be expected to be direction-fixed and horizontal with the fast component of the nystagmus directed towards the intact ear. This paretic direction-fixed type was significantly more common in acute $\mathrm{VN}(80 \%)$ than in follow-up VN (42\%) and MD (26\%), and the recovery direction-fixed type was significantly more common in MD (31\%) than in acute VN (3\%) and follow-up VN (14\%). The recovery direction-fixed type can be explained by the adaptation of vestibular functions, centrally or peripherally, and it was reported that such compensatory changes can occur in patients with minimally damaged canal function $[4,5]$.

We found that direction-changing PN in the head-roll test, which may be attributed to the activation or inhibition of LSCC by otolith particles, was observed in $10 \%, 14 \%$, and $22 \%$ of patients with acute VN, follow-up VN, and MD, respectively. The ratio of geotropic direction-changing PN to apogeotropic direc- 
tion-changing PN was 3:3 in acute $\mathrm{VN}(\mathrm{n}=6), 6: 3$ in follow-up $\mathrm{VN}(\mathrm{n}=9)$, and 7:7 in MD ( $\mathrm{n}=14)$; However, the differences between them were not statistically significant.

Comorbidity of BPPV and MD has been occasionally reported, and the incidence of BPPV in MD varies. While $65 \%$ to $70 \%$ of patients with MD experience BPPV between attacks of MD [6], BPPV was reported to be associated with MD in $8 \%$ to $10 \%$ of MD patients and usually developed $2-5$ years after the onset of MD [7-9]. Although BPPV is more aggressive and intractable when associated with MD $[8,10,11]$, another study showed that the duration of successful treatment of positional vertigo is not different between BPPV in MD and idiopathic BPPV [12]. A high recurrence rate is another distinct characteristic of BPPV secondary to MD $[13,14]$. The maculae of the utricle and saccule may be damaged due to vascular compromise caused by endolymphatic hydrops, and this vascular compromise has been advanced as the causative mechanism linking BPPV to MD [15]. The pathophysiologic correlation between BPPV and MD is further supported by the fact that BPPV developed in the same ear as MD in almost all patients in previous studies $[1,7,12]$.

Degeneration of the vestibular nerve with or without vestibular hair cell damage has been also observed in VN $[16,17]$. Utricular damage may result in otoconial dislodgement leading to BPPV. It was reported that BPPV develops between 2 weeks and 20 years after VN, and seven out of eight patients with PN show direction-changing PN (geotropic in four and apogeotropic in three) [18]. Secondary BPPV has been reported to occur after VN $[12,19,20]$, which may be an important cause of recurrent vertigo in $\mathrm{VN}$ patients during the follow-up period.

Placing vertiginous patients into different positions, provoking unbalanced neural firing from the labyrinths, may cause direction-fixed horizontal PN with the fast component directed towards (paretic) or against (recovery) the intact ear. In VN, the direction-fixed paretic PN was the most common type indicating that a persistent vestibular impairment was augmenting the SN. In $\mathrm{MD}$, direction-fixed recovery $\mathrm{PN}$ was the most common type, suggesting the adaptation of vestibular functions [3-5].

However, direction-changing PN could be also observed in $9 \%-20 \%$ of $\mathrm{VN}$ and MD patients, suggesting the presence of otoliths involving the lateral canal. The affected ear by BPPV can be determined as the side showing more intense nystagmus in the geotropic type and the side showing less intense nystagmus in the apogeotropic type [11,21]. In our study, according to the intensity of the nystagmus in the supine head-roll test, the possible lesioned side of LSCC was not the same to the lesioned ear in seven of 29 patients (24\%) (one of six acute VN patients, one of nine follow-up VN patients, and five of 14 MD patients) (Table 2). There was no significant difference of this discrepancy between the groups. It was reported that the head-roll test could not indicate the affected side correctly in seven $(26.9 \%)$ of 29 lateral canal BPPV patients [21], though there is also a possibili- ty for the BPPV to occur on the unaffected ear.

In the present study, it is difficult to differentiate the LSCC canalolithiasis from light cupula in cases with geotropic type of direction-changing PN because nystagmus was recorded only for 30-60 seconds during a head-roll test and a null plane was not sought to be identified. In addition, the condition of heavy cupula, of which the possibility was addressed in the previous studies, cannot also be ruled out in cases with apogeotropic type of direction-changing PN. As this study utilized retrospective data, we could not obtain any data about the responsiveness to any canalolith repositioning maneuvers or the changes of PN. Once the involved canal is identified, BPPV can be treated with particle repositioning maneuvers moving the otoconia out of the involved canal back to the utricle $[11,22]$. Possibly, the patients with follow-up and MD could have gotten faster improvement if any BPPV was recognized and treated immediately. Another limitation is that Dix-Hallpike maneuver was not included in this study to diagnose a posterior canal BPPV.

In summary, direction-fixed paretic PN indicating a persistent vestibular impairment was the most common type in VN. Direction-fixed recovery $\mathrm{PN}$ was the most common type in MD. However, direction-changing PN, suggesting the presence of otoliths involving the lateral canal, was also observed in $9 \%-$ $20 \%$ of VN and MD patients. Our findings showed that direction-changing and direction-fixed PN could be observed in VN and MD patients and BPPV should be considered, especially when they complain of position-related dizziness. In addition, clinicians should note that direction-changing PN can also be observed in patients with central nervous system disorders such as posterior circulation stroke, neuroinflammation, tumors, and vestibular migraine.

\section{CONFLICT OF INTEREST}

No potential conflict of interest relevant to this article was reported.

\section{ACKNOWLEDGMENTS}

This study was supported by a grant from the Korea Healthcare Technology R\&D Project, Ministry of Health and Welfare, Republic of Korea (HI10C2020).

\section{ORCID}

$\begin{array}{ll}\text { Chang-Hee Kim } & \text { https://orcid.org/0000-0001-5667-861X } \\ \text { Jung Eun Shin } & \text { https://orcid.org/0000-0001-6044-9342 } \\ \text { Myung Hoon Yoo } & \text { https://orcid.org/0000-0002-9158-8924 } \\ \text { Hong Ju Park } & \text { https://orcid.org/0000-0002-6331-8556 }\end{array}$




\section{AUTHOR CONTRIBUTIONS}

Conceptualization: all authors. Data curation \& Formal analysis: CHK, HJP.Writing - original draft, review \& editing: all authors.

\section{REFERENCES}

1. Strupp M, Brandt T. Vestibular neuritis. Semin Neurol. 2009 Nov; 29(5):509-19.

2. Kim CH, Shin JE, Kim TS, Shim BS, Park HJ.Two-dimensional analysis of head-shaking nystagmus in patients with Meniere's disease. J Vestib Res. 2013 Jan;23(2):95-100.

3. Kim CH, Shin JE, Song CI, Yoo MH, Park HJ. Vertical components of head-shaking nystagmus in vestibular neuritis, Meniere's disease and migrainous vertigo. Clin Otolaryngol. 2014 Oct;39(5):261-5.

4. Horan B, Dillon P, Faverdin P, Delaby L, Buckley F, Rath M. The interaction of strain of Holstein-Friesian cows and pasture-based feed systems on milk yield, body weight, and body condition score. J Dairy Sci. 2005 Mar;88(3):1231-43.

5. Park H, Shin J, Jeong Y, Kwak H, Lee Y. Lessons from follow-up examinations in patients with vestibular neuritis: how to interpret findings from vestibular function tests at a compensated stage. Otol Neurotol. 2009 Sep;30(6):806-11.

6. Paparella MM. Benign paroxysmal positional vertigo and other vestibular symptoms in Meniere disease. Ear Nose Throat J. 2008 Oct; 87(10):562

7. Baloh RW, Honrubia V, Jacobson K. Benign positional vertigo: clinical and oculographic features in 240 cases. Neurology. 1987 Mar; 37(3):371-8

8. Gross EM, Ress BD, Viirre ES, Nelson JR, Harris JP. Intractable benign paroxysmal positional vertigo in patients with Meniere's disease. Laryngoscope. 2000 Apr;110(4):655-9.

9. Gananca CF, Caovilla HH, Gazzola JM, Gananca MM, Gananca FF. Epley's maneuver in benign paroxysmal positional vertigo associated with Meniere's disease. Braz J Otorhinolaryngol. 2007 Jul-Aug; 73(4):506-12.

10. Perez N, Martin E, Zubieta JL, Romero MD, Garcia-Tapia R. Benign paroxysmal positional vertigo in patients with Meniere's disease treated with intratympanic gentamycin. Laryngoscope. 2002 Jun; 112(6):1104-9.

11. Song CI, Kang BC, Yoo MH, Chung JW, Yoon TH, Park HJ. Management of 210 patients with benign paroxysmal positional vertigo: AMC protocol and outcomes. Acta Otolaryngol. 2015 May;135(5): 422-8.

12. Lee NH, Ban JH, Lee KC, Kim SM. Benign paroxysmal positional vertigo secondary to inner ear disease. Otolaryngol Head Neck Surg. 2010 Sep;143(3):413-7.

13. Balatsouras DG, Ganelis P, Aspris A, Economou NC, Moukos A, Koukoutsis G. Benign paroxysmal positional vertigo associated with Meniere's disease: epidemiological, pathophysiologic, clinical, and therapeutic aspects. Ann Otol Rhinol Laryngol. 2012 Oct;121(10): 682-8.

14. Dornhoffer JL, Colvin GB. Benign paroxysmal positional vertigo and canalith repositioning: clinical correlations. Am J Otol. 2000 Mar;21(2):230-3.

15. Karlberg M, Hall K, Quickert N, Hinson J, Halmagyi GM. What inner ear diseases cause benign paroxysmal positional vertigo? Acta Otolaryngol. 2000 Mar;120(3):380-5.

16. Schuknecht HF, Kitamura K. Second Louis H. Clerf lecture. Vestibular neuritis. Ann Otol Rhinol Laryngol Suppl. 1981 Jan-Feb;90(1 Pt 2):1-19.

17. Nadol JB Jr. Vestibular neuritis. Otolaryngol Head Neck Surg. 1995 Jan;112(1):162-72.

18. Harada K, Oda M, Yamamoto M, Nomura T, Ohbayashi S, Kitsuda C. A clinical observation of benign paroxysmal positional vertigo (BPPV) after vestibular neuronitis (VN). Acta Otolaryngol Suppl. 1993;113(Suppl 503):61-3.

19. Mandala M, Santoro GP, Awrey J, Nuti D. Vestibular neuritis: recurrence and incidence of secondary benign paroxysmal positional vertigo. Acta Otolaryngol. 2010 May;130(5):565-7.

20. Kim YH, Kim KS, Kim KJ, Choi H, Choi JS, Hwang IK. Recurrence of vertigo in patients with vestibular neuritis. Acta Otolaryngol. 2011 Nov;131(11):1172-7.

21. Choung YH, Shin YR, Kahng H, Park K, Choi SJ. 'Bow and lean test' to determine the affected ear of horizontal canal benign paroxysmal positional vertigo. Laryngoscope. 2006 Oct;116(10):1776-81.

22. Bhattacharyya N, Baugh RF, Orvidas L, Barrs D, Bronston LJ, Cass S, et al. Clinical practice guideline: benign paroxysmal positional vertigo. Otolaryngol Head Neck Surg. 2008 Nov;139(5 Suppl 4):S47-81. 